\title{
Capillary Electrophoresis Separations of Bundled and Individual Carbon Nanotubes
}

\author{
Stephen K. Doorn,*\$\$ Michael S. Strano, ${ }^{\#}$ Michael J. O'Connell, ${ }^{\#}$ Erik H. Haroz, \\ Kristy L. Rialon," Robert H. Hauge," and Richard E. Smalley" \\ Chemistry Division (MS-J563, C-ACS), Los Alamos National Laboratory, Los Alamos, NM, 87545, and \\ Department of Chemistry and Center for Nanoscale Science and Technology, Rice University, \\ Houston, Texas 77251-1892
}

Received: December 19, 2002; In Final Form: April 1, 2003

\begin{abstract}
Capillary electrophoresis (CE) has been performed on polymer-stabilized bundles and sodium dodecyl sulfate (SDS) suspensions of high-pressure carbon monoxide (HiPco)-produced single-walled carbon nanotubes. Realtime Raman spectroscopy has been used for detection and characterization of the separation process. Radialbreathing-mode vibrational frequencies are sensitive to nanotube diameter. Monitoring of these modes demonstrates the separation of poly(vinylpyrrolidone) (PVP)-stabilized tubes into fractions of differing bundle composition. Separations on SDS suspensions indicate that CE is capable of separating bundled nanotubes from isolated individuals on the basis of differences in nanotube mobility, depending on changes in diameter or bundle size.
\end{abstract}

\section{Introduction}

Fulfilling the promise that carbon nanotubes hold for new lightweight, high-performance materials and applications in molecular electronics and sensors, ${ }^{1}$ among other areas, will require production of bulk quantities of pure material. Catalytic gas-phase growth methods ${ }^{2}$ are beginning to prove themselves for large-scale production of raw nanotube material. There has also been impressive progress in purifying as-produced material to remove unwanted catalyst particles and amorphous carbon material. ${ }^{3-9}$ With the increasing availability of relatively pure product, it is becoming feasible to pursue geometry-selective separations of carbon nanotubes.

Current purified samples contain a broad length and diameter distribution with a corresponding mixture of electronic properties. Differences in the geometrical parameters $(n, m)$ will determine whether the nanotubes are conducting or semiconducting. ${ }^{10}$ Changes in $(n, m)$ also result in differences in diameter. A $(10,6)$ tube will be semiconducting and have a diameter of $1.11 \mathrm{~nm}$, whereas a $(11,11)$ tube is conducting, with a diameter of $1.51 \mathrm{~nm}$. Thus, the ability to separate tubes on the basis of geometry will ultimately also provide a degree of selectivity over electronic properties. Field-flow fractionation $(\mathrm{FFF})^{11}$ and size-exclusion chromatography ${ }^{12,13}$ have demonstrated some success at length-based separations but result in fractions with broad length distributions. Recent work using flow field-flow fractionation, however, demonstrates progress in producing narrower distributions. ${ }^{14}$ Electrophoretic separation is also beginning to look promising. Gel electrophoresis has produced fractions with similar length distributions as those found for FFF separations. ${ }^{15}$ It has recently been demonstrated that capillary electrophoresis (CE) is capable of producing highresolution separations of carbon nanotubes that are based on differences in length. ${ }^{16}$ The bulk of the electric-arc-produced nanotubes used in that initial study were expected to fall within

* Author to whom correspondence should be addressed. E-mail: skdoorn@ lanl.gov.

\$ Los Alamos National Laboratory.

\# Rice University. a limited diameter range, and, thus, diameter selectivity in the separations was not expected to be apparent. However, the proposed mechanism for CE separations ultimately depends on charge-density differences between tubes of differing geometries, as well as differences in mobility. Because the total charge on the nanotubes is expected to be proportional to their surface area, charge-density differences ultimately will be dependent on diameter. Thus, CE may be a viable method for producing diameter-dependent separations of nanotubes.

In the work presented here, we perform $\mathrm{CE}$ on samples that contain a broad distribution of nanotube diameters. The ability to monitor diameter-dependent vibrational spectra through Raman detection of the separation process allows us to observe composition-dependent changes in nanotube fractions over the course of a separation. We find that $\mathrm{CE}$ is capable of separating polymer-stabilized nanotube bundles into fractions of differing composition and can also separate bundled nanotubes from isolated individuals. These results suggest that CE may have the potential for producing diameter-selective separations.

\section{Experimental Section}

Single-walled carbon nanotubes (SWNTs) were produced using the high-pressure carbon monoxide (HiPco) method. ${ }^{2}$ Poly(vinylpyrrolidone) (PVP)-suspended tubes $(1.4 \mathrm{~g} / \mathrm{L})$ were produced as described previously. ${ }^{15}$ The nanotubes used for the PVP suspension were initially purified by gas-phase oxidation, hydrochloric acid extraction, and high-temperature annealing. ${ }^{15}$ Atomic force microscopy (AFM) images of the PVP samples show them to have a length distribution ranging from $50 \mathrm{~nm}$ to $1 \mu \mathrm{m}$. The PVP samples contain nanotube bundles with a distribution of composition and bundle size. Sodium dodecyl sulfate (SDS) suspensions ( $2 \mathrm{~g} / \mathrm{L})$ were prepared by sonicating untreated, raw solid nanotubes in $0.5 \%$ SDS solution for 10 min at $80 \mathrm{~W}$ with a $50 \%$ duty cycle, using a horn sonicator.

CE was performed on a lab-built system, using a $75-\mu \mathrm{m}-$ inside-diameter, 75-cm-long fused silica capillary with detection at the midpoint. Capillaries were conditioned for $3 \mathrm{~min}$ with $0.2 \mathrm{M} \mathrm{NaOH}$, followed by flushing with deionized water and 


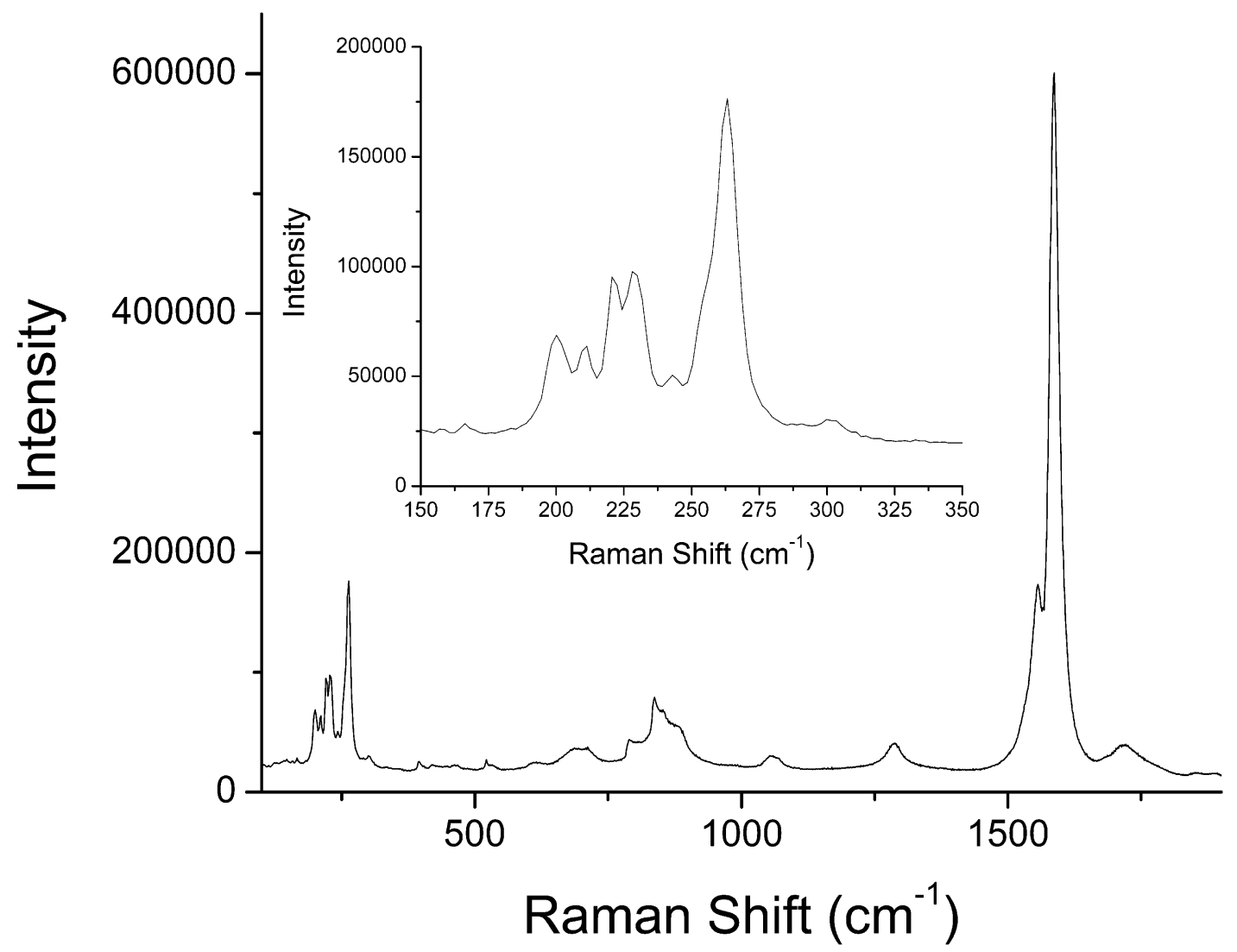

Figure 1. Raman spectrum (20 mW at $785 \mathrm{~nm}$ excitation, 1-s integration) of bulk solid HiPco-produced nanotubes. Inset: Radial breathing mode (RBM) region of bulk solid.

then run buffer before each separation. Separations were run with an applied voltage of $+15 \mathrm{kV}$, using $50 \mathrm{mM}$ trizma base as a run buffer $(15 \mathrm{kV}$ was below the limit of linear behavior in a voltage-versus-current plot for our buffer systems). Buffers for separations of the SDS suspensions also contained $0.5 \%$ SDS. Samples were pressure-introduced at 500 mbar for $2 \mathrm{~s}$ by pulling sample into a $2-\mathrm{mL}$ void volume with a syringe attached to the effluent end of the capillary.

Raman detection was performed using 20-mW, 785-nm diode laser excitation through a fiber-optic probe head that incorporated a $20 \times, 0.5$ N.A. objective for sample illumination and $180^{\circ}$ collection of Raman scatter through a window burned into the capillary coating. Scattered light was collected into a Kaiser Optical HoloSpec $f / 1.8$ imaging spectrograph. An entrance slit $50 \mu \mathrm{m}$ wide resulted in a spectral resolution of $4 \mathrm{~cm}^{-1}$. Detection was accomplished with a back-illuminated redsensitive Princeton Instruments CCD camera $(100 \times 1340$ pixels), using integration times of $1 \mathrm{~s}$. Full spectra were collected continuously in real time during the course of a separation.

\section{Results}

Behavior of PVP-Stabilized Nanotubes. A bulk Raman spectrum of the purified solid HiPco-produced nanotubes is shown in Figure 1. Of particular interest for our studies is the low-frequency region of the spectrum (Figure 1, inset), which is dominated by the nanotube radial breathing mode (RBM) vibrations. The observed frequencies are inversely proportional to the diameter of the nanotubes ${ }^{17}$ and range from 169 to 373 $\mathrm{cm}^{-1}$ in our sample, which translates to a diameter distribution ranging from 0.6 to $1.3 \mathrm{~nm}$. The seven most-intense bands $\left(207-267 \mathrm{~cm}^{-1}\right)$ correspond to diameters of $0.88-1.15 \mathrm{~nm}$. The presence of a broad range of observable diameters in the HiPco- produced samples makes them attractive for investigating the potential of CE for diameter selectivity.

A representative electropherogram from a separation of PVPsuspended sample is shown in Figure 2a. By monitoring the intensity of the strong $1591 \mathrm{~cm}^{-1}$ nanotube Raman band, we find that nanotubes first appear $\sim 17$ min into a separation. (Note that each point in the electropherogram actually represents a complete Raman spectrum.) Except for the appearance of a few spikes (bands limited in width to the integration time of $1 \mathrm{~s}$ ), the electropherogram is dominated by primarily broad, incompletely resolved fractions. This domination is in contrast to the behavior observed in the original work on SDS-dissolved nanotubes by Doorn et al., ${ }^{16}$ in which well-resolved, extremely narrow fractions were observed. The time of the first appearance of nanotubes is reproducible from run to run, as are the positions of the major features in the electopherogram. However, the relative intensities of these features can change between runs. This variation may be due to minor differences in the manual sampling method and also possibly results from the small and potentially heterogeneous sampling volume $(\sim 100-300 \mathrm{~nL})$. The presence of distinct bands in the electropherogram indicates that some separation process is occurring; however, the question of whether this is due only to the previously observed lengthbased mechanism ${ }^{16}$ or combines a diameter-based process requires further analysis of the Raman spectra to determine.

Closer inspection of how the RBM region of the Raman spectra changes over the course of a separation will provide information on whether diameter-selective separations are occurring superimposed on the length-based mechanism. Electropherograms showing changes in the intensity ratios of the $267 \mathrm{~cm}^{-1}$ band, relative to the $234 \mathrm{~cm}^{-1}$ band, and of the 234 $\mathrm{cm}^{-1}$ band, relative to the $207 \mathrm{~cm}^{-1}$ band, are shown in Figures 


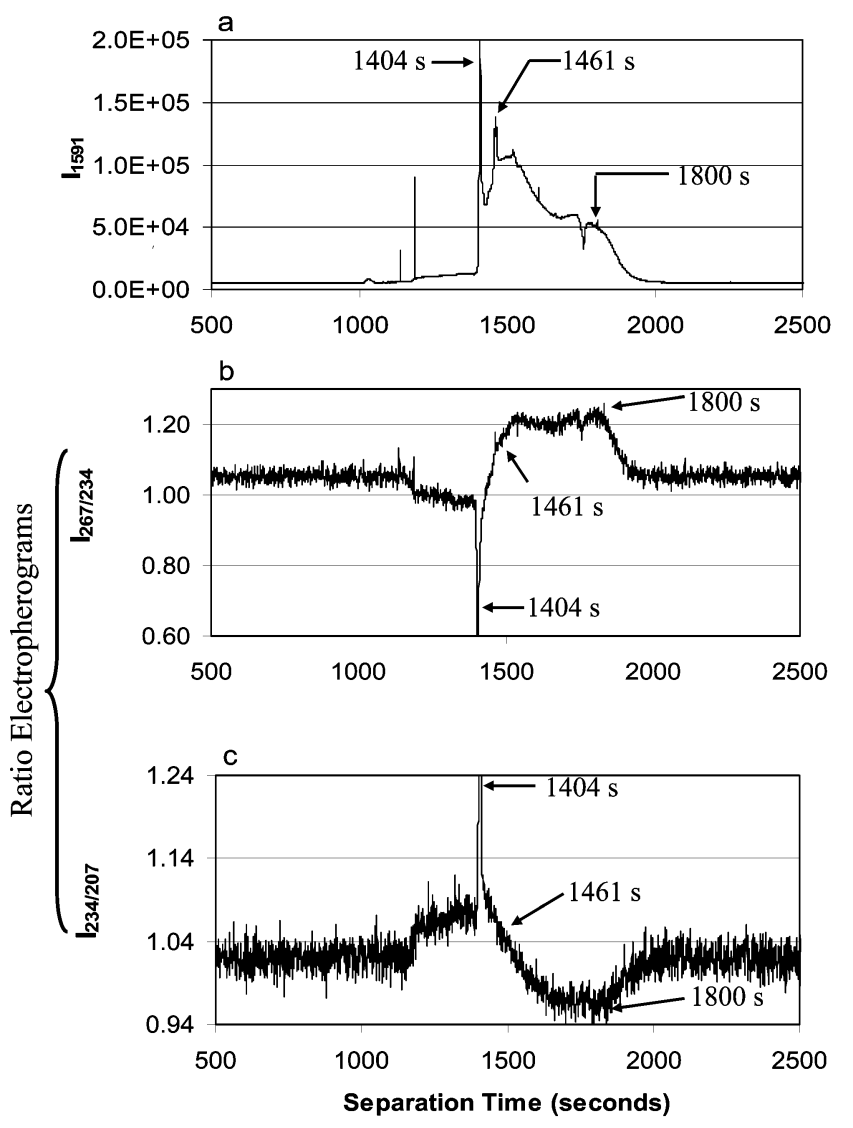

Figure 2. Electropherograms of PVP-stabilized nanotubes monitoring (a) the $1591 \mathrm{~cm}^{-1}$ mode intensity, (b) the intensity ratio of the 267 $\mathrm{cm}^{-1}$ to $234 \mathrm{~cm}^{-1}$ modes, and (c) the intensity ratio of the $234 \mathrm{~cm}^{-1}$ to $207 \mathrm{~cm}^{-1}$ modes.

$2 \mathrm{~b}$ and c, respectively. At early times (1200-1450 s), the band at $234 \mathrm{~cm}^{-1}$ is the most prominent, with a significant increase in intensity occurring at $1404 \mathrm{~s}$. At intermediate times, the 267 $\mathrm{cm}^{-1}$ band is strongest. Finally, from $\sim 1600 \mathrm{~s}$ to the end of the separation, the $234 \mathrm{~cm}^{-1}$ mode diminishes in intensity.

Differences in selected spectra within these regions are shown for the highlighted times in Figure 3 and are also compared to the bulk, unseparated solution spectrum. Nanotubes observed at intermediate times display spectra (Figure 3c) very similar to that observed for the bulk solution (Figure 3a). At early times, the $234 \mathrm{~cm}^{-1}$ mode is clearly dominant, whereas the $267 \mathrm{~cm}^{-1}$ band is diminished, with respect to all other modes present. At the end of the separation (Figure 3d), all vibrations are weaker overall in intensity, but the intermediate frequencies (215-234 $\mathrm{cm}^{-1}$ ) are significantly weakened, compared to the 207 and 267 $\mathrm{cm}^{-1}$ modes.

Behavior of SDS-Suspended Nanotubes. A typical electropherogram for the SDS-suspended nanotube sample is shown in Figure 4. A series of baseline-separated, sharp and intense spikes (widths limited to the 1-s integration time) are observed throughout the early part of the separation (Figure 4a). These results are similar to those found by Doorn et al. in their separations of SDS-suspended arc-produced nanotubes. ${ }^{16}$ Closer inspection of the electropherogram, however, reveals that the sharp spikes are on top of a broad background (Figure 4b) that more resembles the results obtained for the PVP suspended tubes.

As seen in the ratio electropherogram of the $267 \mathrm{~cm}^{-1}$ intensity divided by the $234 \mathrm{~cm}^{-1}$ intensity (Figure 4c), the behavior of the RBMs undergoes large changes over the course of the separation. Three distinct regions of behavior are

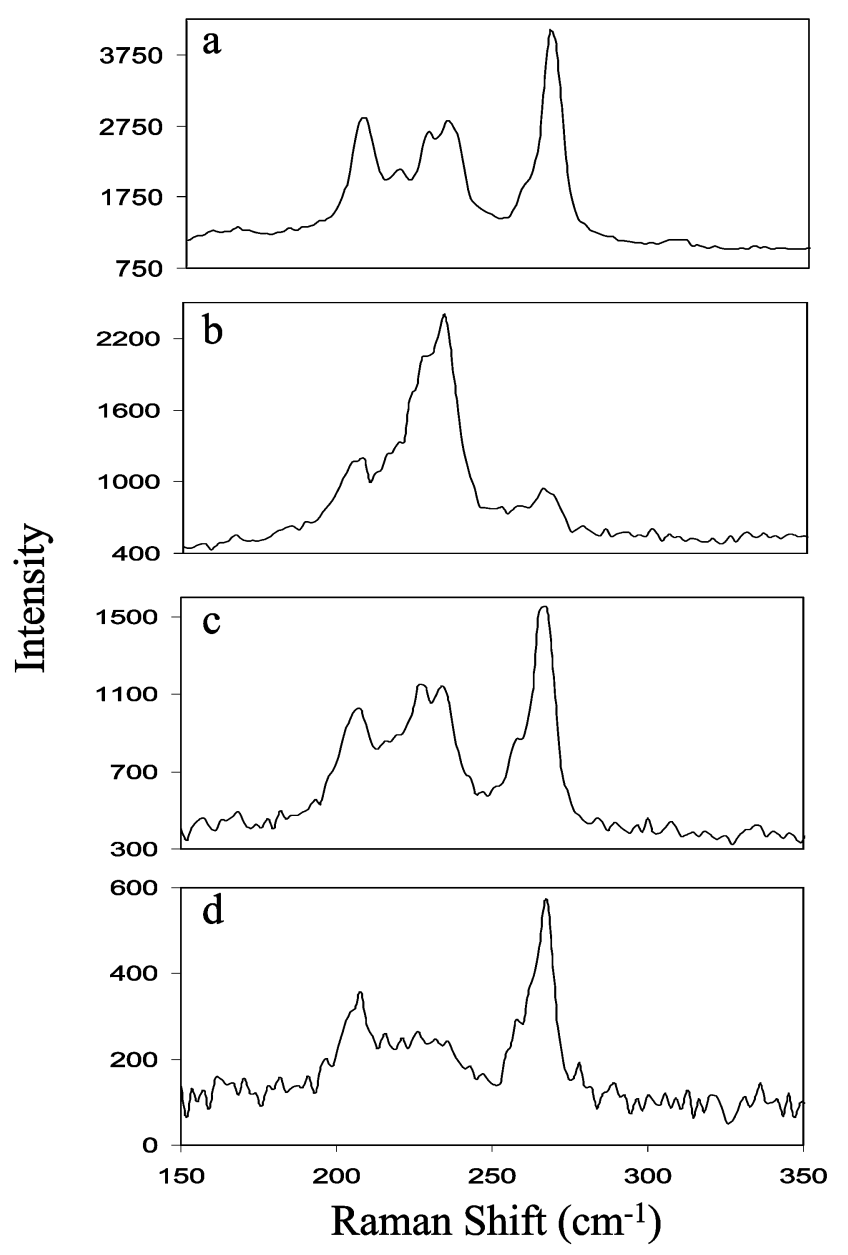

Figure 3. (a) Radial breathing mode (RBM) Raman spectrum of bulk, unseparated PVP-stabilized nanotube solution. Selected Raman spectra from separation shown in Figure 2 are also presented: (b) spectrum at $1404 \mathrm{~s}$, (c) spectrum at $1461 \mathrm{~s}$, and (d) spectrum at $1800 \mathrm{~s}$.

observed. In the first region, ranging from the first appearance of nanotubes to $\sim 500 \mathrm{~s}$, ratios for both the spikes and the broad envelope are greater than the baseline ratio observed before the appearance of nanotubes. This observation indicates that spectra in this region are dominated by the $267 \mathrm{~cm}^{-1}$ mode. At intermediate times (region 2, 500-677 s), the intense spikes are still positive going, but the broad envelope begins to trend to values less than the baseline. At longer times (region 3, from $678 \mathrm{~s}$ to the end of the separation), the intense spikes disappear and the broad envelope remains in the region below the baseline. This later-time region is dominated by the $234 \mathrm{~cm}^{-1}$ mode throughout the broad envelope, as well as for the weak spikes observed in this region. The first appearance of nanotubes occurs reproducibly after $\sim 5 \mathrm{~min}$ into a separation. The observed positions of individual spikes and relative intensities of the major features change from run to run, because of sampling heterogeneities. However, the positions and duration of the three regions observed in the ratio electropherogram are reproducible.

Representative spectra for these three regions are compared to the bulk solution spectrum in Figure 5. A spectrum of a spike at $482 \mathrm{~s}$ is shown in Figure $5 \mathrm{~b}$. The spectrum is dominated by the $267 \mathrm{~cm}^{-1}$ band and is similar to that for the bulk solution. In the spike spectrum, however, the relative intensity near 234 $\mathrm{cm}^{-1}$ is $\sim 50 \%$ of that in the bulk spectrum. The spectrum at $482 \mathrm{~s}$ is identical to all spikes observed in the first two regions of the electropherogram, except for variations in intensity. The broad background envelope spectra in this region are also 

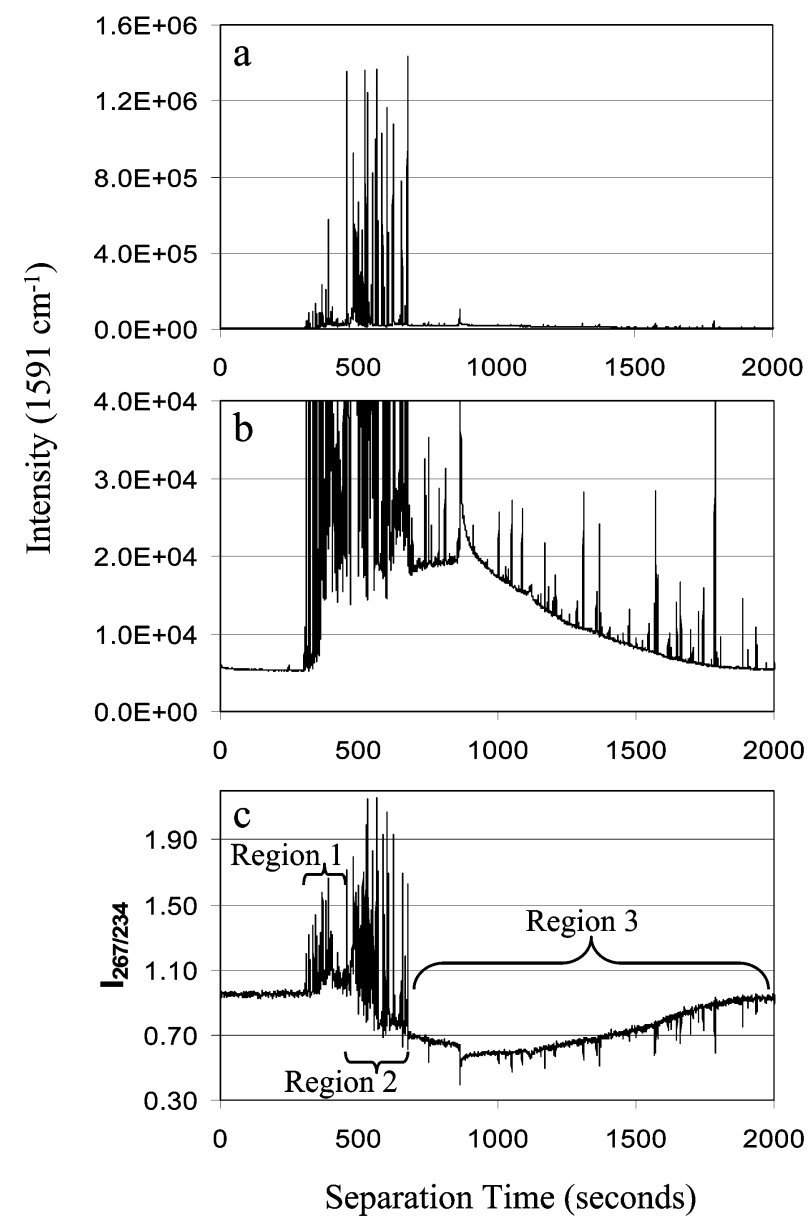

Figure 4. (a) Electropherogram of SDS-suspended nanotubes, monitoring the $1591 \mathrm{~cm}^{-1}$ intensity. (b) Electropherogram showing expansion of the $y$-axis. (c) Electropherogram monitoring the intensity ratio of the $267 \mathrm{~cm}^{-1}$ to $234 \mathrm{~cm}^{-1}$ modes.

identical to this spike-the observed frequencies and relative intensities remain the same. In the second electropherogram region (500-677 s), the spike spectra also display the same frequencies and relative intensities as those that are observed in the first region. However, the background envelope now shifts to spectra that are dominant in the $234 \mathrm{~cm}^{-1}$ mode, with its intensity being approximately twice that of the $267 \mathrm{~cm}^{-1}$ band. The spectrum taken at $633 \mathrm{~s}$ (Figure 5c) is representative of this second region. A further dramatic shift in the relative intensities is seen when one goes into the third region. Almost all the RBM intensity is now in the $234 \mathrm{~cm}^{-1}$ mode, plus two shoulders located at 226 and $215 \mathrm{~cm}^{-1}$ (Figure 5d). Spectra in this region are also similar to those observed in the fraction at $1404 \mathrm{~s}$ in the PVP separations (Figure 3b), although with lower intensity observed in the $267 \mathrm{~cm}^{-1}$ mode. Low-intensity spikes are also observed in this third region. Spectra for these spikes are quite different than those found for the strong spikes in the first region. Rather than being dominant in the $267 \mathrm{~cm}^{-1}$ mode, the spectra are quite similar to the envelope spectra observed in the second region (Figure 5c), with the $234 \mathrm{~cm}^{-1}$ band being approximately twice the intensity of that at $267 \mathrm{~cm}^{-1}$.

\section{Discussion}

The variations in the RBM intensities indicate that there are diameter-dependent spectral changes occurring over the course of a separation. It is tempting to relate these intensity changes directly to changes in the relative population of the corresponding diameter tube. However, there are several complicating

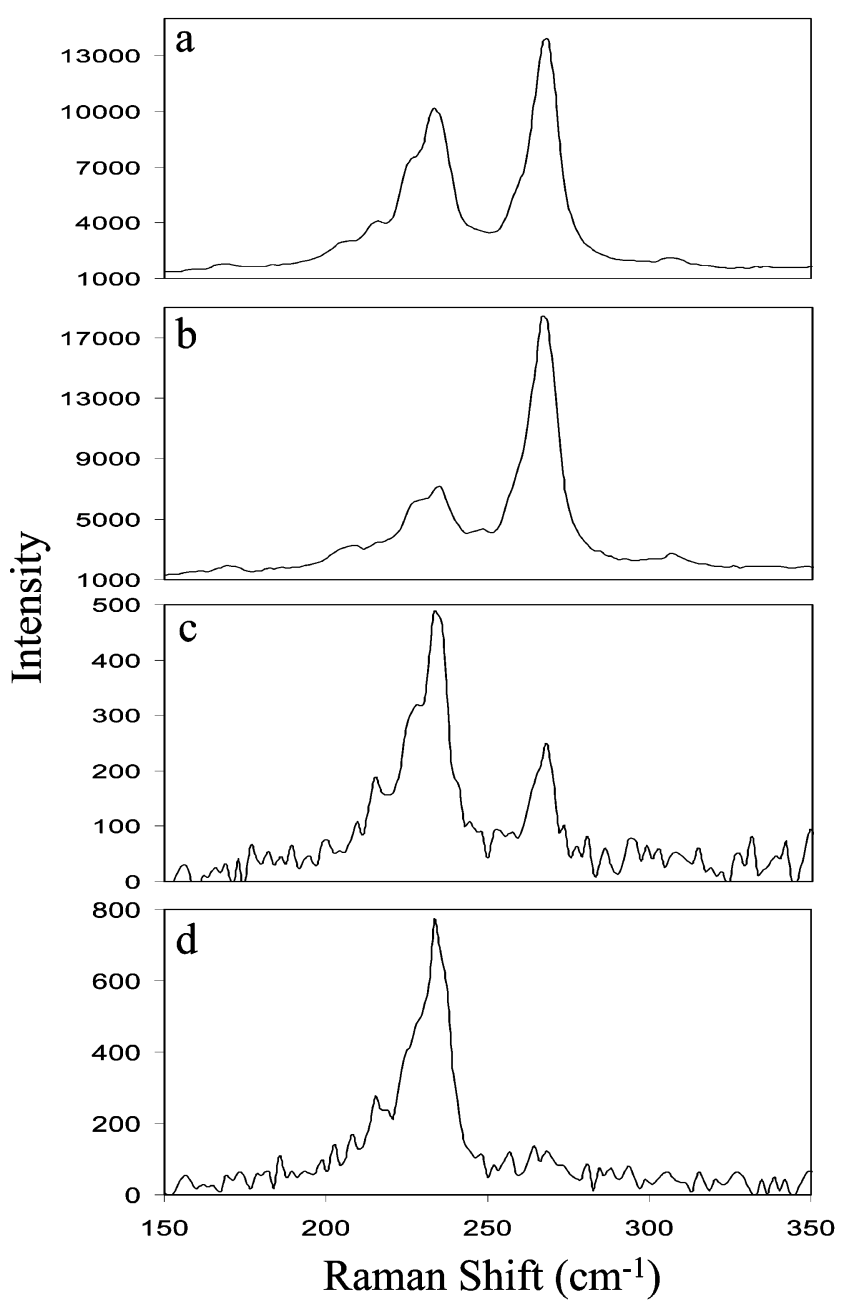

Figure 5. (a) Radial breathing mode (RBM) Raman spectrum of bulk unseparated SDS-suspended nanotubes. Selected Raman spectra from separation shown in Figure 4 are also presented: (b) spectrum at 482 $\mathrm{s}$, (c) spectrum at $633 \mathrm{~s}$, and (d) spectrum at $918 \mathrm{~s}$.

factors. The PVP samples used in this investigation are known to be bundles that vary in size and composition. Thus, the behavior observed is not that for separations of isolated, individual nanotubes. Furthermore, Raman spectra for nanotubes display strongly diameter-dependent resonance enhancement effects. Spectra for nanotubes of differing diameter will be enhanced to varying degrees for a fixed excitation wavelength. This is an important consideration for both the PVP and SDS systems. Only RBMs for nanotubes with a van Hove singularity near the $785 \mathrm{~nm}$ excitation energy will be significantly enhanced.

These enhancement effects will be further perturbed in the PVP samples, because of the strong interaction expected among nanotubes in a given bundle. Bundling (roping) of the nanotubes gives rise to an orthogonal energy dispersion to the onedimensional density of states found in individual nanotubes, resulting in a change in energy spacing of the van Hove singularities. ${ }^{18,19}$ For semiconducting nanotubes, the absorption maxima are broadened and shifted to lower energy. ${ }^{19,20}$ This is a particularly strong effect for the $267 \mathrm{~cm}^{-1}$ mode, which can be used as an indicator of the degree of roping. For sufficiently large bundles, the electronic resonance for this mode strongly overlaps the $785 \mathrm{~nm}$ excitation energy. As the bundle size decreases, the resonance energy shifts to shorter wavelengths. This shift will result in a decrease in resonance Raman intensity at $267 \mathrm{~cm}^{-1}$ to a minimum when overlap is minimized between the $785 \mathrm{~nm}$ excitation energy and the electronic resonance for 


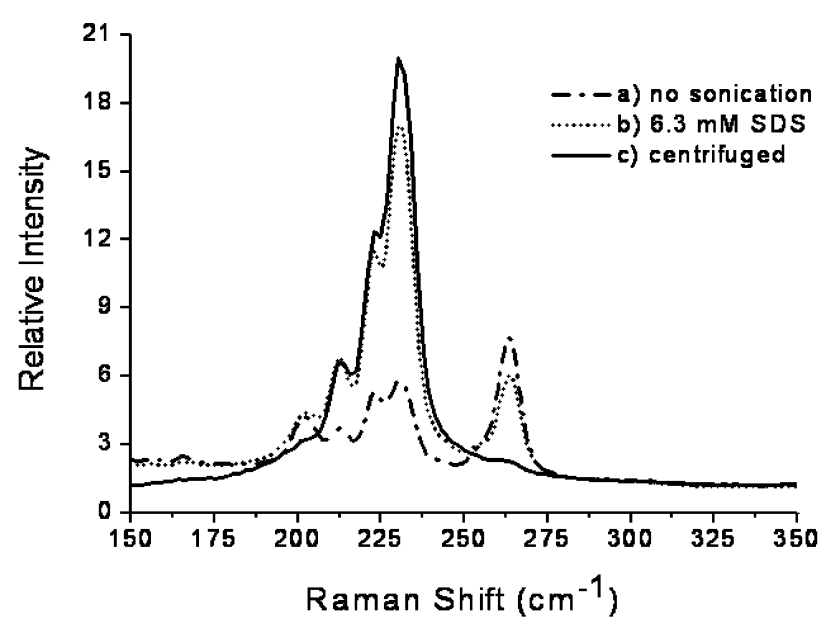

Figure 6. Radial breathing mode (RBM) spectra for (a) an aqueous slurry of roped nanotubes, (b) a sonicated sample of nanotubes in 6.3 $\mathrm{mM}$ SDS, and (c) isolated individuals collected via centrifugation (ref 20). Note: the absolute intensities for spectra $a$ and $b$ are directly comparable; an arbitrary scaling factor was applied to spectrum c.

an individual nanotube. This trend is clearly seen in Figure 6. The $267 \mathrm{~cm}^{-1}$ mode loses intensity when one goes from a purely roped sample, through a sonicated mixture of roped and isolated nanotubes, and finally to a sample of purely isolated individuals. Conversely, the $234 \mathrm{~cm}^{-1}$ mode gains in intensity when one goes from a roped sample to isolated individuals. Resonance Raman excitation profiles on samples of isolated individuals show the $234 \mathrm{~cm}^{-1}$ mode to be strongly enhanced with $785 \mathrm{~nm}$ excitation. Thus, enhancement effects and perturbations may result in spectra that do not accurately indicate the dominant nanotube type in a given population but may be useful in indicating the relative nanotube bundle size. Perturbations of enhancement behavior are not expected to be dependent on nanotube length for our samples.

The bundle size/cross section for a collection of nanotubes is defined by the number of nanotubes present and by their individual diameters. The results for the PVP samples demonstrate that we can separate a sample into fractions of differing composition, that exhibit differing electronic resonance behavior. The majority of PVP-stabilized bundles will contain a random distribution of the available tube diameters. Therefore, spectra observed over the course of a separation might be expected to be similar to that observed for the bulk solution. This condition is observed at intermediate times (Figure 3c). Even at very early and late times, when the ratio electropherograms are showing some enhancement of particular RBMs (Figure 2b, c), the spectra are still similar to the bulk spectrum. The somewhatreduced relative intensity of the $267 \mathrm{~cm}^{-1}$ mode found in the region from 1100 to $1400 \mathrm{~s}$ (Figure 2b) suggests that these bundles may be smaller than those eluting at later times. The sharp loss in intensity of the $234 \mathrm{~cm}^{-1}$ mode at late times (Figure $3 \mathrm{~d}$ ) may be due to a decrease in the population of corresponding diameter tubes. Alternatively, the bundle compositions at this point in the separation may yield a shift of the $234 \mathrm{~cm}^{-1}$ resonance energy that results in poor overlap with the excitation wavelength.

The most-dramatic changes in the spectra are observed for the sharp, intense fraction occurring at $1404 \mathrm{~s}$ (Figure 3b). Although the dominance of the $234 \mathrm{~cm}^{-1}$ mode in this fraction may mean that the corresponding diameter of the nanotube is being enriched in this fraction, this seems improbable. A more likely explanation is that the bundle size for this fraction is significantly smaller than that found in the remainder of the sample, which would result in a significant loss in intensity in the $267 \mathrm{~cm}^{-1}$ mode, as is observed for this fraction.

The degree to which bundles of different composition exist in a fraction is indicated by the relative intensity and width of that fraction. For those that represent a more random distribution of diameters, there should be a small and continuous variation in cross section/size across the range of composition. This variation will result in relatively broad bands, as observed in Figure 2a. Areas in which significant enrichment of one combination or diameter of nanotubes occurs should yield a narrower distribution of total cross section/size. This will result in relatively narrow, intense fractions. This is best illustrated for the fraction occurring at $1404 \mathrm{~s}$. The growth and decay of this fraction occurs over a period of just $20 \mathrm{~s}$, followed by the much broader fraction containing spectral distributions similar to the bulk solution. In addition to broadening from a variation in cross section or size distributions, all fractions will be further broadened because of the superposition of separations that are based on length differences. This will also cause some overlap of bands that might otherwise represent "pure" compositions. Thus, the observed spectra will not be pure indicators of composition.

Significant changes in RBM spectra over the course of a separation are also observed for the SDS-suspended samples (Figures 4c, 5). On considering the loss in relative intensity in the $234 \mathrm{~cm}^{-1}$ mode in the first-region spectra, compared to that for the bulk solution (Figure 5a, b), it seems likely that this population is recovered in the spectra observed in the second and third regions (Figure 5c, d). The presence of three different regions of spectral behavior demonstrates that nanotube fractions of at least three different electronic behavior are being separated. As with the PVP samples, however, one cannot simply say that the shift in the relative intensities for the different RBMs is due to a change in the absolute population of different diameter nanotubes. Changes in resonance enhancement conditions and interaction effects are also likely.

Although the $267 \mathrm{~cm}^{-1}$ mode is almost absent in the third separation region (Figures 4c, 5d), some weak intensity is still clearly present. This suggests that, rather than elimination of the diameter tube corresponding to the $267 \mathrm{~cm}^{-1}$ band altogether, the observed intensity differences may instead be due to a change in resonance excitation conditions for this fraction. Therefore, changes in the relative intensities of the 267 and 234 $\mathrm{cm}^{-1}$ modes throughout the separation are likely due to the same effects that are observed and discussed for the PVP-suspended systems. The spectra observed in the first region (Figures 4c, $5 b)$ are likely due to large aggregates/ropes of nanotubes, because the spectra are dominated by the $267 \mathrm{~cm}^{-1}$ "roping" mode. The weaker intensity observed for this mode in the second region (Figure 5c) suggests that aggregates present during this time are significantly smaller. Finally, the near loss of intensity of this mode in the third region (Figure 5d) suggests that nanotubes present at these times are aggregates of minimum size or are individual isolated nanotubes exhibiting minimum interaction with other tubes in solution. The Raman intensity observed from the spikes (in some cases, as high as 100 times greater than that observed for the isolated tubes) also supports the conclusion that they are due to aggregates. Strong signals may result from a significant number of tubes being present per aggregate. Weaker intensities observed in the second and third separation regions are consistent with these nanotubes being smaller aggregates and individual nanotubes. Finally, there is a good comparison between spectra of known aggregates and 

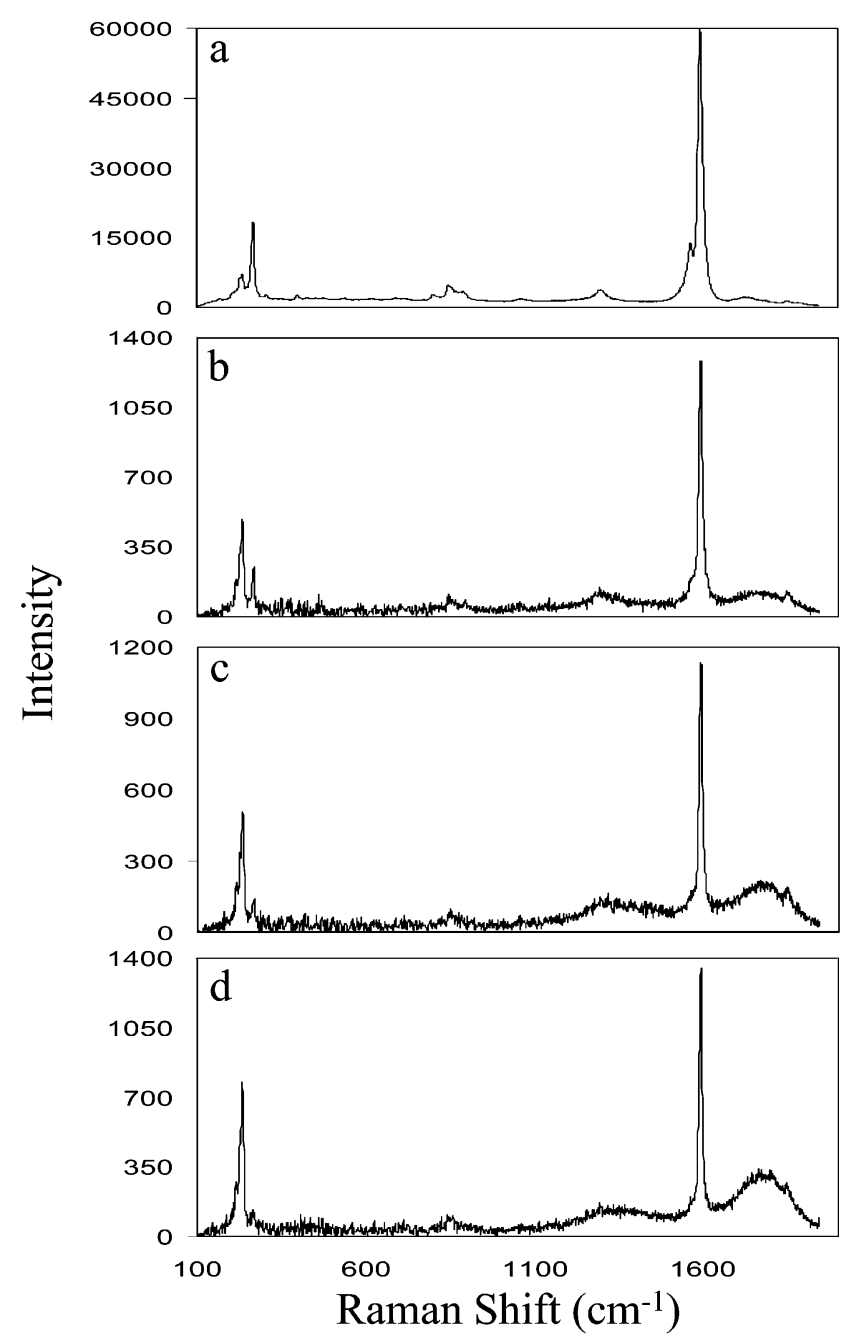

Figure 7. Full Raman spectra from the separation shown in Figure 4: (a) spectrum at $482 \mathrm{~s}$, (b) spectrum at $633 \mathrm{~s}$, (c) spectrum at $809 \mathrm{~s}$, and (d) spectrum at $918 \mathrm{~s}$.

isolated individuals (Figure 6a, c) and their proposed counterparts from the separations.

Additional evidence for these assignments can be found by examining the complete spectra for the fractions. By comparing spectra for the three regions (Figure 7), we find that the latetime spectra (Figure 7c, d) contain fluorescence features that are absent in the earlier regions. These fluorescence bands occur on either side of the $1591 \mathrm{~cm}^{-1}$ band, with absolute wavelengths for emission of 878 and $915 \mathrm{~nm}$. These emission bands have only recently been discovered for SWNTs by O'Connell et al. ${ }^{20}$ The bands have been assigned as band-gap emission from the first van Hove transitions that occur in semiconducting tubes. The key to their discovery was the ability to produce truly isolated individual nanotubes in solution. These authors found that the aggregation of tubes quenched the fluorescence, because of the presence of increased energy-decay pathways or through direct interaction with metallic tubes in a given bundle.

Fluorescence is not observed at any point in the separation of the PVP samples, which is consistent with the previously discussed findings that it can only be observed in isolated tubes. However, the processing used for the PVP samples could lead to isolated nanotubes that do not fluoresce. The oxidation step used in their purification will eliminate fluorescence through the introduction of a large number of defect sites on the nanotube sidewalls. If isolated tubes are present in the PVP sample, their concentration is low, because the RBM intensity patterns expected for isolated nanotubes are not observed. The closest candidate for this behavior would be the nanotubes in the fraction at $1404 \mathrm{~s}$ (Figure 2). However, as previously discussed, the RBM intensities observed for this fraction (Figure $3 b$ ) are more consistent with a small bundle rather than for an isolated individual. Low concentrations of isolated individuals would be expected for this sample, as a consequence of the PVP suspension procedure. The centrifugal concentration step results in a high degree of roped tubes being present in the PVP sample. ${ }^{15,20}$

The findings for the SDS-suspended samples strongly suggest that spectra observed for early-to-intermediate times in Figure 4 (the first two separation regions), particularly in the strong spikes, are from aggregated nanotubes. This observation also suggests that the strong spikes observed by Doorn et al. ${ }^{16}$ are probably also due to aggregates. Observation of fluorescence in the third separation region indicates that it is dominated by isolated individual nanotubes. Spikes observed in this region, although still dominant in the $234 \mathrm{~cm}^{-1} \mathrm{RBM}$, do not contain the fluorescence bands and are, therefore, assigned as small aggregates. The large number of aggregate fractions observed in the SDS separations is likely a result of the relatively mild sonication conditions used for sample preparation. This may also be a contributing factor in run-to-run differences in the appearance time of specific fractions. Roping of the nanotubes will be a dynamic process, resulting in changes in aggregate composition and size between samplings for separate CE runs.

The information contained in the observed fluorescence bands emphasizes again that the Raman spectra can be misleading, in regard to the composition of a given fraction. The fluorescence bands at 878 and $915 \mathrm{~nm}$ correspond to nanotubes of diameter 0.69 and $0.76 \mathrm{~nm}$, respectively. ${ }^{21}$ Nanotubes of this diameter will have RBMs at 336 and $306 \mathrm{~cm}^{-1}$, respectively. These bands are found in spectra for the aggregate spikes (Figure 5b); however, because of weak resonance enhancement at $785 \mathrm{~nm}$ excitation for these individual nanotubes, they are not observed in the third separation region. However, the observation of their fluorescence bands clearly indicates their presence in this fraction. Nanotubes of certain diameters may be present in a given fraction, yet not be observed, because of spectral silence arising from poor resonance enhancement.

The separations observed in the SDS system build on the PVP results. In addition to being able to separate fractions with differing electronic behaviors, fractions that differ in large-scale geometry can also be obtained. The SDS results demonstrate that bundles of different cross-sectional area can be separated from each other, and that bundles can be separated from individual isolated nanotubes. However, the fact that the fraction of isolated nanotubes in the SDS separations contains a wide range of diameters suggests that the separations may not strictly be a function of nanotube diameter or bundle cross section. Instead, the molecular weight of the species in a fraction may be the important mechanistic factor for separations, although molecular weight will ultimately depend on bundle cross section or individual diameter. The fact that separations depend on many factors is evident in the overlap observed between the three regions in the SDS separations. Large aggregate spikes continue to be observed in the region containing primarily small to moderate-sized aggregates, whereas these smaller aggregates continue to appear as spikes throughout the region containing primarily isolated individual nanotubes. The potential for such overlapping behaviors was recognized in the original work on length-dependent separations ${ }^{16}$ and will be an important problem to overcome in future $\mathrm{CE}$ work on carbon nanotubes. 


\section{Conclusion}

The results of this work indicate that capillary electrophoresis (CE) has the potential to separate carbon nanotubes, not only by differences in length (as demonstrated previously ${ }^{16}$ ) but also by differences in size or other geometric factors, such as diameter or cross section. We have shown, for poly(vinylpyrrolidone) (PVP)-stabilized tube bundles, that separations result in fractions containing bundles of different electronic properties and may also be dependent on variations in bundle size. CE on sodium dodecyl sulfate (SDS) suspensions can separate large aggregates from smaller bundles and produce a relatively pure fraction of individual isolated nanotubes. Separation of the aggregates from individual nanotubes may be based on differences in molecular weight or differences in cross section/ diameter. These results are also significant in that they are the first independent confirmation of the ability of semiconducting carbon nanotubes to emit band-gap fluorescence. Our results were obtained in a completely different approach than what resulted in the original fluorescence discovery (separation based on differences in density ${ }^{20}$ ). Both results indicate that sonication is capable of producing individual isolated nanotubes and that this capability is critical to gaining a complete understanding of fundamental nanotube properties. Our results also underscore the importance of recognizing resonance enhancement effects when interpreting Raman data from a given set of nanotubes. Shifts in resonance enhancement must be weighed against the possibility of changes in sample composition when explaining variations in the relative intensity.

The results on the SDS suspensions also indicate that the full potential of CE for diameter-selective separations may best be reached through working with samples of fully isolated individual tubes. Bulk production of these samples is just now becoming feasible,${ }^{20}$ and separations of them will be the subject of future work. These types of separations will be important, not only for producing samples consisting of a narrow range of geometrical and electronic properties, but also for making the study of these pure properties on isolated individual nanotubes possible, without the need to go to the single nanotube level.

Acknowledgment. Research at Los Alamos National Laboratory has been supported by internal LDRD funding. Research at Rice University has been supported by the National Science Foundation (NSF) (CHE-9900417), the NSF Focused Research Group on Fullerene Nanotube Chemistry (DMR-0073046), the NSF Center for Biological and Environmental Nanotechnology (EEC-0118007), and the Robert A. Welch Foundation (C-0689 and C-0807). Support from NASA (NCC 9-77) for the development of the HiPco method is also gratefully acknowledged.

\section{References and Notes}

(1) Dresselhaus, M. S., Dresselhaus, G., Avouris, P., Eds. Carbon Nanotubes: Synthesis, Structure, Properties, and Applications; Springer: Berlin, 2001; Vol. 80 .

(2) Nikolaev, P.; Bronikowski, M. J.; Bradley, R. K.; Rohmund, F.; Colbert, D. T.; Smith, K. A.; Smalley, R. E. Chem. Phys. Lett. 1999, 313, 91.

(3) Rinzler, A. G.; Liu, J.; Dai, H.; Nikolaev, P.; Huffman, C. B.; Rodriguez-Macias, F. J.; Boul, P. B.; Lu, A. H.; Heymann, D.; Colbert, D. T.; Lee, R. S.; Fischer, J. E.; Rao, A. M.; Eklund, P. C.; Smalley, R. E. Appl. Phys. A: Mater. Sci. Process. 1998, 67, 29.

(4) Dillon, A. C.; Gennet, T.; Jones, K. M.; Alleman, J. L.; Parilla, P. A.; Heben, M. J. Adv. Mater. 1999, 11, 1354

(5) Li, F.; Cheng, H. M.; Xing, Y. T.; Tan, P. H.; Su, G. Carbon 2000, 38, 2041.

(6) Yudasaka, M.; Zhang, M.; Jabs, C.; Iijima, S. Appl. Phys. A: Mater. Sci. Process. 2000, 71, 449 .

(7) Coleman, J. N.; Dalton, A. B.; Curran, S.; Rubio, A.; Davey, A. P.; Drury, A.; McCarthy, B.; Lahr, B.; Ajayan, P. M.; Roth, S.; Barklie, R. C.; Blau, W. J. Adv. Mater. 2000, 12, 213.

(8) Holzinger, M.; Hirsch, A.; Bernier, P.; Duesberg, G. S.; Burghard, M. Appl. Phys. A: Mater. Sci. Process. 2000, 70, 599.

(9) Niyogi, S.; Hu, H.; Hamon, M. A.; Bhomik, P.; Zhao, B.; Rozenzhak, S. M.; Chen, J.; Itkis, M. E.; Meier, M. S.; Haddon, R. C. J. Am. Chem. Soc. 2001, 123, 733 .

(10) Dresselhaus, M. S.; Dresselhaus, G.; Pimenta, M. Eur. Phys. J. D 1999, 9,69

(11) Liu, J.; Rinzler, A. G.; Dai, H.; Hafner, J. H.; Bradley, R. K.; Boul, P. J.; Lu, A.; Iverson, T.; Shelimov, K.; Huffman, C. B.; Rodriguez-Macias, F.; Shon, Y.-S.; Lee, T. R.; Colbert, D. T.; Smalley, R. E. Science 1998, $280,1253$.

(12) Duesberg, G. S.; Muster, J.; Krstic, V.; Burghard, M.; Roth, S. Appl. Phys. A: Mater. Sci. Process. 1998, 67, 117.

(13) Duesberg, G. S.; Blau, W.; Byrne, H. J.; Muster, J.; Burghard, M.; Roth, S. Synth. Met. 1999, 103, 2484.

(14) Chen, B.; Selegue, J. P. Anal. Chem. 2002, 74, 4774

(15) O'Connell, M. J.; Boul, P.; Ericson, L. M.; Huffman, C.; Wang, Y.; Haroz, E.; Kuper, C.; Tour, J.; Ausman, K. D.; Smalley, R. E. Chem. Phys. Lett. 2001, 342, 265.

(16) Doorn, S. K.; Fields, R. E., III; Hu, H.; Hamon, M. A.; Haddon, R. C.; Selegue, J. P.; Majidi, V. J. Am. Chem. Soc. 2002, 124, 3169.

(17) Rao, A. M.; Richter, E.; Bandow, S.; Chase, B.; Eklund, P. C.; Williams, K. A.; Fang, S.; Subbaswamy, K. R.; Menon, M.; Thess, A.; Smalley, R. E.; Dresselhaus, G.; Dresselhaus, M. S. Science 1997, 275, 187.

(18) Rao, A. M.; Chen, J.; Richter, E.; Schlecht, U.; Eklund, P. C.; Haddon, R. C.; Venkateswaran, U. D.; Kwon, Y.-K.; Tomanek, D. Phys. Rev. Lett. 2001, 86, 3895.

(19) Reich, S.; Thomsen, C.; Ordejon, P. Phys. Rev. B 2002, 65, 5411.

(20) O'Connell, M. J.; Bachilo, S. M.; Huffman, C. B.; Moore, V. C.; Strano, M. S.; Haroz, E. H.; Rialon, K. L.; Boul, P. J.; Noon, W. H.; Kittrell, C.; Ma, J.; Hauge, R. H.; Weisman, R. B.; Smalley, R. E. Science 2002, 297, 593.

(21) Bachilo, S. M.; Strano, M. S.; Kittrell, C.; Hauge, R. H.; Smalley, R. E.; Weisman, R. B. Science 2002, 298, 2361. 